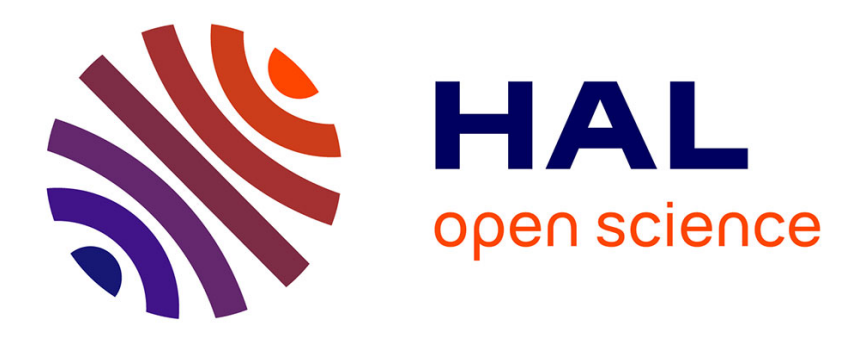

\title{
Relativistic Electron Generation in Nonuniform Underdense Plasmas
}

G. Manfredi, R. Bingham, R. Dendy

\section{To cite this version:}

G. Manfredi, R. Bingham, R. Dendy. Relativistic Electron Generation in Nonuniform Underdense Plasmas. Journal de Physique IV Proceedings, 1995, 05 (C6), pp.C6-93-C6-96. 10.1051/jp4:1995618 . jpa-00253981

\section{HAL Id: jpa-00253981 https://hal.science/jpa-00253981}

Submitted on 1 Jan 1995

HAL is a multi-disciplinary open access archive for the deposit and dissemination of scientific research documents, whether they are published or not. The documents may come from teaching and research institutions in France or abroad, or from public or private research centers.
L'archive ouverte pluridisciplinaire HAL, est destinée au dépôt et à la diffusion de documents scientifiques de niveau recherche, publiés ou non, émanant des établissements d'enseignement et de recherche français ou étrangers, des laboratoires publics ou privés. 


\title{
Relativistic Electron Generation in Nonuniform Underdense Plasmas
}

\author{
G. Manfredi, R. Bingham and R.O. Dendy \\ Rutherford Appleton Laboratory, Chilton, Didcot, Oxon, OX11, OQX, U.K.
}

\begin{abstract}
Simulations of relativistic electron generation by stimulated Raman scattering are presented. The effect of a nonuniform plasma density, such as may occur in a laser fusion scenario, is investigated. A simple WKB theory accounts for the wavenumber shifts in the spectra.
\end{abstract}

\section{INTRODUCTION}

In laser-plasma acceleration experiments at low density, measurements of $\mathrm{MeV}$ electrons have been made $[1,2]$ and attributed to resonant acceleration by large amplitude Langmuir waves nonlinearly excited by the laser beams. In laser fusion, considerable attention has focussed on indirect drive schemes using hohlraum targets where the laser beams ablate the inside wall of a hohlraum creating a hot plasma. During this phase parametric instabilities can occur, in particular stimulated Raman instabilities producing waves that may accelerate energetic electrons [3]. In addition, a density gradient is often present in a typical hohlraum experiment, since the plasma is created by ablation of the inside wall, and its density decreases from the wall to the interior of the hohlraum. To simulate wave excitation and electron acceleration in these scenarios, the combined effect of various types of instability is studied here using a 1-D Eulerian Vlasov code $[4,5]$.

\section{THE PHYSICAL MODEL}

We consider a linearly polarized electromagnetic wave propagating along the $x$ direction, described by the electric field $E_{y}$ and the magnetic field $B_{z}$. The wave interacts with a plasma, in which all quantities are taken to be functions of the longitudinal coordinate $x$ and of time only. In our onedimensional model the electron plasma is described by the relativistic Vlasov equation for $f\left(x, p_{x}, t\right)$ :

$$
\frac{\partial f}{\partial t}+\frac{p_{x}}{\gamma m} \frac{\partial f}{\partial x}-e\left(E_{x}+u_{y} B_{z}\right) \frac{\partial f}{\partial p_{x}}=0
$$

where $\gamma=\left(1+p_{x}^{2} / m^{2} c^{2}\right)^{\frac{1}{2}}$ and $p_{x}=m \gamma u_{x}$. Since we are interested in short time scales compared to the ion plasma period, the ions are taken to be immobile. The transverse velocity $u_{y}$, generated by the electromagnetic field of the laser, is non-relativistic and obeys

$$
\frac{\partial u_{y}}{\partial t}=-\frac{e}{m} E_{y}
$$

This expresses conservation of the transverse canonical momentum $p_{y}=u_{y}-(\mathrm{e} / \mathrm{m}) A_{y}$, with $E_{y}=$ 
$-\partial A_{y} / \partial t$. The longitudinal electric field is obtained self-consistently from Poisson's equation,

$$
\frac{\partial^{2} \phi}{\partial x^{2}}=-\frac{e}{\epsilon_{0}}\left[n_{i}(x)-n_{e}(x, t)\right]
$$

with $n_{e}=\int f d p_{x}$ and $E_{x}=-\partial \phi / \partial x$. The transverse electromagnetic fields obey Maxwell's equations,

$$
\begin{gathered}
\frac{\partial B_{z}}{\partial t}=-\frac{\partial E_{y}}{\partial x} \\
\frac{\partial E_{y}}{\partial t}=-c^{2} \frac{\partial B_{z}}{\partial x}-\frac{1}{\epsilon_{o}} J_{y} \\
J_{y}=-c n_{e}(x, t) u_{y}(x, t)
\end{gathered}
$$

The laser wave enters the computational box from the left side, and encounters the plasma profile shown in Fig 1. Initially, the electron distribution function is a relativistic double Maxwellian with two temperatures, with $5 \%$ of the total population in the hot tail.

\section{NUMERICAL RESULTS}

By writing the pertinent equations in dimensionless form, three dimensionless quantities appear to play a crucial role: $\omega_{o} / \omega_{p}$, the ratio of laser to plasma frequency; $v_{t h} / c$, the electron thermal velocity; and $v_{o s c} / c=e E_{o} / m \omega_{o} c$, the "quiver" velocity, where $E_{o}$ is the laser electric field. Since, in our case, the plasma frequency is a function of position, we take as a reference $\omega_{p o}$, the value of the plasma frequency at the left boundary, corresponding to the density $n=n_{0}$. In the simulations, these parameters take the values $\omega_{o} / \omega_{p o}=2.97, v_{o s c} / c=0.11, v_{t h} / c=0.15$ in the cold bulk of the plasma, and $v_{t h} / c=0.25$ in the hot tail. The corresponding value of $k_{o}$ is $2.8 \omega_{p o} c^{-1}$ and the total length of the plasma is $L=224.4 \mathrm{c}_{p}{ }^{-1}$. It is readily shown that the plasma is undercritical with respect to forward Raman scattering (i.e. $n / n_{0}<4$ ) in the region $x<0.6 L=122 c \omega_{p o}{ }^{-1}$. However, since $\omega_{0}>\omega_{p}$ everywhere, the laser wave can penetrate even in the region forbidden to the excited plasma wave.

The plot of the electrostatic field (in units of $m \omega_{0} c / e$ ) at the end of the simulation (Fig 2) shows that the plasma wave decays exponentially in the region where the plasma is overcritical. The corresponding wavenumber spectrum is given in Fig 3 , showing a broad peak around $2 \omega_{p o} c^{-1}$. Figs 4 and 5 show respectively the spectra of the backscattered and forward scattered laser light. In a uniform plasma, one expects to observe Stokes and anti-Stokes liglit as sidebands of the pump wave at $k_{o} \pm \omega_{p o} c^{-1}$ in the forward scattered spectrum, and a plasma wave at $k_{p}=\omega_{p o} c^{-1}$. However, due to inhomogeinities, these wavenumber will experience a shift. Since the density varies slowly compared to the laser wavelength, a. WKB approximation holds, in which one makes use of the local value of the plasma frequency to derive the conditions for resonance. A simple calculation yields the following expressions for the pump, Stokes and anti-Stokes waves :

$$
\begin{gathered}
\frac{c^{2} k_{0}^{2}}{\omega_{p o}^{2}}=\frac{\omega_{0}^{2}}{\omega_{p o}^{2}}-1-\frac{\delta n}{n_{0}} \\
\frac{c^{2} k_{S, A S}^{2}}{\omega_{p o}^{2}}=\frac{\omega_{0}}{\omega_{p o}}\left(\frac{\omega_{0}}{\omega_{p o}} \mp 2 \mp \frac{\delta n}{n_{0}}\right)
\end{gathered}
$$

Here $\delta n / n_{o}=\left(n-n_{0}\right) / n_{0}$ increases from zero at the left boundary to $\simeq 1.12$ at $x=0.6 L$ where the density becomes critical. Choosing $\delta n / n_{0}=0.8$, we are able to recover with relatively good 


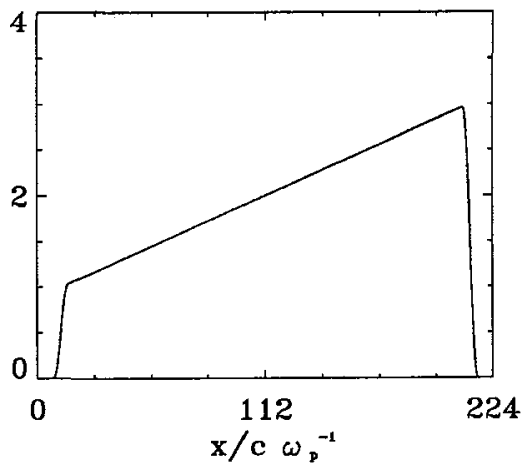

Fig 1 : Density profile

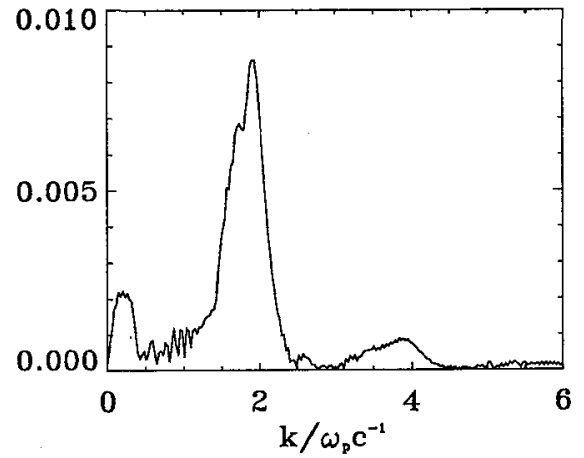

Fig 3 : Electrostatic field spectrum

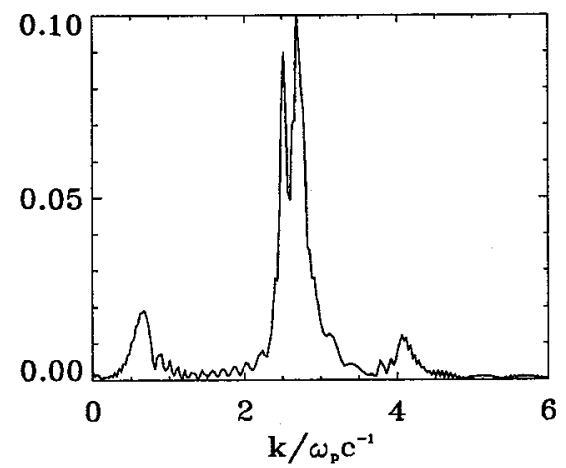

Fig 5 : Forward electromagnetic spectrum

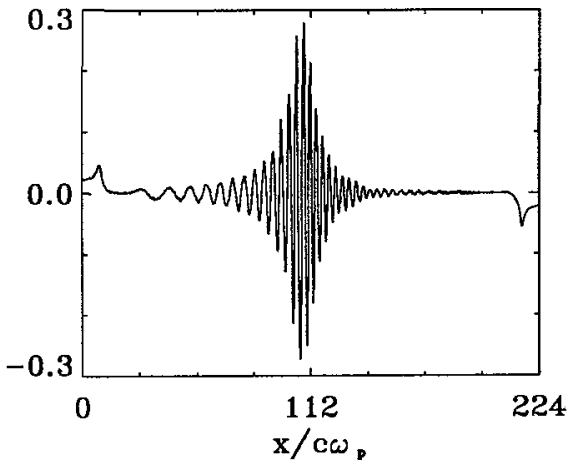

Fig 2 : Electrostatic field

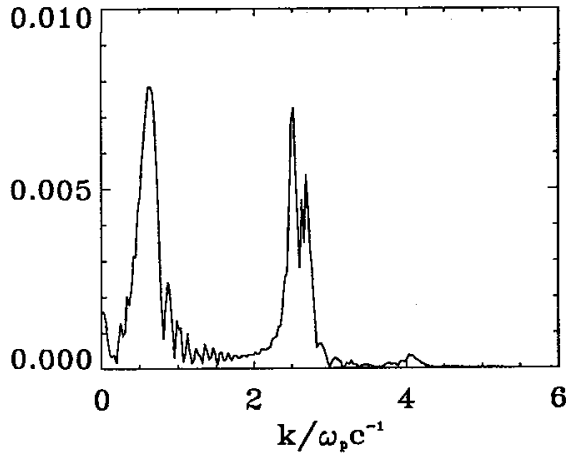

Fig 4 : Backward electromagnetic spectrum

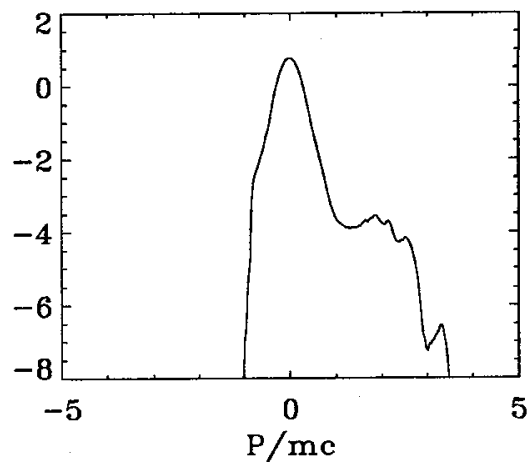

Fig 6 : Momentum distribution function 
approximation the wavenumbers appearing in the spectra shown in Figs 2-4. In fact, Eqs 6 and 7 yield for this value : $k_{0}=2.65 \omega_{p o} c^{-1} ; k_{S}=0.71 \omega_{p o} c^{-1}$ and $k_{A S}=4.14 \omega_{p o} c^{-1}$. The corresponding plasma waves have wavenumbers : $k_{p}=k_{o}-k_{S}=1.94 \omega_{p o} c^{-1}$ and $k_{p}=k_{A S}-k_{o}=1.49 \omega_{p o} c^{-1}$. The backscattered light with $k_{B}=-0.71 \omega_{p o} c^{-1}$ couples with the pump wave to give rise to the plasma wave $k_{p}=k_{o}-k_{B}=3.36 \omega_{p o} c^{-1}$, which is slightly smaller than the observed value. The backscattered light with $k_{B}=-2.65 \omega_{p o} c^{-1}$ would excite a plasma wave of wavenumber $2 k_{o}$, which is however heavily Landau damped $\left(2 k_{o} \lambda_{D} \simeq 0.8\right)$ and does not appear in the spectrum. Finally, the peak in the electrostatic spectrum at $\simeq 0.25 \omega_{p o} c^{-1}$ roughly matches the width of the plasma wave envelope. The acceleration of electrons to relativistic velocities is shown in Fig 6 by plotting the distribution function, integrated in space between $x=L / 2$ and $x=3 L / 4$, at the end of the simulation. Energies up to $1.5 \mathrm{MeV}$ are obtained, in the presence of a laser field of intensity $2.5 \times 10^{16} \mathrm{~W} \mathrm{~cm}^{-2}$ in a plasma density $n_{0}=10^{19} \mathrm{~cm}^{-3}$.

In conclusion, we have produced numerical results of electron acceleration in a nonuniform plasma by stimulated Raman scattering. When the density gradient is small compared to the laser wavelength, a WKB estimate gives the correct wavenumbers, provided a suitable "effective" density is used. Different density profiles could yield a more complex behaviour.

\section{Acknowledgements}

The authors are grateful to A Ghizzo, P Bertrand, E Fijalkow and M Shoucri for providing the numerical code. This work was supported in part by the Commission of the European Communities under Contract ERBCHBICT941009.

\section{References}

[1] Joshi C, Mori W B, Katsouleas T, Dawson J M, Kindel J M and Forslund D W, Nature 311 (1984) 525.

[2] Modena A et al., Laser and Particle Beams (1995) submitted.

[3] Decker C D, Mori W B and Katsouleas T, Phys Rev E50 (1994) 3338.

[4] Ghizzo A, Bertrand P, Shoucri M, Fijalkow E and Feix M R J Comp Phys 108 (1993) 105.

[5] Manfredi G, Bingham R and Dendy R O, Laser and Particle Beams (1995) submitted. 